\title{
Matrix approach to hypercomplex Appell polynomials*
}

\author{
Lidia Aceto $^{\mathrm{a}}$, Helmuth R. Malonek ${ }^{\mathrm{b}}$, and Graça Tomaz ${ }^{\mathrm{c}}$ \\ ${ }^{a}$ Department of Mathematics, University of Pisa, Italy \\ b Department of Mathematics, University of Aveiro, Portugal \\ ${ }^{\mathrm{c}}$ Department of Mathematics, Polytechnic Institute of Guarda, \\ Portugal
}

August 28, 2018

\begin{abstract}
Recently the authors presented a matrix representation approach to real Appell polynomials essentially determined by a nilpotent matrix with natural number entries. It allows to consider a set of real Appell polynomials as solution of a suitable first order initial value problem. The paper aims to confirm that the unifying character of this approach can also be applied to the construction of homogeneous Appell polynomials that are solutions of a generalized Cauchy-Riemann system in Euclidean spaces of arbitrary dimension. The result contributes to the development of techniques for polynomial approximation and interpolation in non-commutative Hypercomplex Function Theories with Clifford algebras.
\end{abstract}

Keyword: hypercomplex differentiability, Appell polynomials, creation matrix, Pascal matrix

MSC: 65F60, 30G35, 11B83.

* This work was supported by Portuguese funds through the CIDMA-Center for Research and Development in Mathematics and Applications, and the Portuguese Foundation for Science and Technology ("FCT-Fundação para a Ciência e Tecnologia"), within project PEst-OE/MAT/UI4106/2014. 


\section{Introduction}

In [1] the authors presented a matrix representation approach to all types of Appell polynomial sequences $\left\{p_{k}(x)\right\}_{k \geq 0}$ of one real variable which relies essentially on the matrix $H$ defined by

$$
(H)_{i j}= \begin{cases}i, & i=j+1 \\ 0, & \text { otherwise }, \quad i, j=0,1, \ldots, m .\end{cases}
$$

In fact, considering that a sequence of real polynomials of degree $k\left\{p_{k}(x)\right\}_{k \geq 0}$ is called Appell polynomial sequence if

$$
\frac{d}{d x} p_{k}(x)=k p_{k-1}(x), \quad k=1,2, \ldots,
$$

cf. [3], by introducing the vector $\mathbf{p}(x)=\left[\begin{array}{llll}p_{0}(x) & p_{1}(x) & \cdots & p_{m}(x)\end{array}\right]^{T}$, from relation (2) we obtain the first order differential equation

$$
\frac{d}{d x} \mathbf{p}(x)=H \mathbf{p}(x)
$$

whose general solution is

$$
\mathbf{p}(x)=e^{H x} \mathbf{p}(0) .
$$

It is evident that the role of $H$ is twofold: it acts both as derivation matrix and also as some type of creation matrix; in fact, the different kinds of Appell polynomials are uniquely determined from (4) by choosing the entries of the initial value vector $\mathbf{p}(0)$. In some sense, this matrix reveals the arithmetical tape which glues the different types of Appell polynomials together.

The prototype of an Appell sequence are the monomials $p_{k}(x)=x^{k}$. The Bernoulli polynomials as the most prominent representatives of Appell polynomials are in Numerical Analysis almost so important as orthogonal polynomials are [12, 13, 25]. But the list of Appell polynomials includes, among others, also the classical polynomials named after Frobenius-Euler, Hermite, Laguerre, and Chebyshev.

Although an old subject, during the last two decades the interest in Appell polynomials and their applications has significantly increased. As very few examples, recent applications of Appell polynomials exist in fields like probability theory and statistics, cf. [2, 28], linear elasticity [4] or approximation of 3D-mappings in [23]. Results in the framework of noncommutative Clifford algebras and related to the Gel'fand-Tsetlin branching approach gave evidence to Appell polynomial sequences with shift, cf. [26], 
as sequences of orthogonal polynomials in several variables, cf. [6, 20]. Operational approaches based on Appell polynomials for generalizing Jacobi, Laguerre, Gould-Hopper, and Chebyshev polynomials are used in the recent papers [7, 8, 10]. Employing methods of representation theory, they are also tools for applications in quantum physics 31 .

Some authors were concerned with finding new characterizations of Appell polynomials themselves through new approaches. We mention, for instance, the approach developed in [30], which makes use of the generalized Pascal functional matrices and the characterization proposed in [14] which is based on a determinantal definition. As previously quoted, the authors introduced in [1] a matrix approach in the real case which has already been applied in the context of "image synthesis" [27, and in connection with "evolution equations" for the construction of a new algorithm of deflation [29].

In addition, Appell polynomial sequences were subject to innumerable generalizations, mostly depending from the type of applications where they could be advantageously used, including multivariate commutative or noncommutative settings.

This paper intends to confirm the advantageously use of the matrix approach to real Appell polynomial sequences developed in [1] for the case of monogenic polynomials in arbitrary dimensions and in a noncommutative hypercomplex setting, i.e. for Clifford algebra valued polynomials in the kernel of a generalized Cauchy-Riemann operator, cf. [15]. In [19] it has been shown that monogenic functions are hypercomplex differentiable. Therefore the classical definition in (2) by the derivative property can be analogously used for defining monogenic Appell sequences with respect to the hypercomplex derivative, cf. [5, 16, 17, 24].

The paper is organized as follows. Basic concepts and notations used in Hypercomplex Function Theory are given in Section 2. In Section 3 the extension to the hypercomplex case of the unifying matrix approach developed in [1] is introduced. Since the hypercomplex generalized CauchyRiemann operator is a linear combination of two different types of first order differential operators (a scalar one and a vector operator in the sense of the underlying Clifford algebra) also two different nilpotent matrices $H$ and $\tilde{H}$ are involved. Their relationship is essential for guaranteeing the desired Appell property. The relation between $H$ and $\tilde{H}$ reveals in a new and condensed matrix form the transition from the real to the hypercomplex case. Applying the results of Section 3 and the concept of a transfer matrix, in Section 4 some of the main hypercomplex monogenic counterparts of real Appell sequences are listed. 


\section{Basic concepts and notation}

For an independent reading, in this section we repeat briefly some notions and results useful in the sequel, mainly following [9, 15, 18, 22.

Definition 2.1. Let $\left\{e_{1}, e_{2}, \ldots, e_{n}\right\}$ be an orthonormal basis of the Euclidean vector space $\mathbb{R}^{n}$ provided with a non-commutative product according to the multiplication rules $e_{i} e_{j}+e_{j} e_{i}=-2 \delta_{i j}, i, j=1,2, \ldots, n$, where $\delta_{i j}$ is the Kronecker symbol. The associative $2^{n}$-dimensional Clifford algebra $\mathcal{C} \ell_{0, n}$ over $\mathbb{R}$ is the set of numbers $\alpha \in \mathcal{C} \ell_{0, n}$ of the form $\sum_{A} \alpha_{A} e_{A}$, with the basis $\left\{e_{A}: A \subseteq\{1, \ldots, n\}\right\}$ formed by $e_{A}=e_{h_{1}} e_{h_{2}} \ldots e_{h_{r}}, 1 \leq h_{1}<\cdots<$ $h_{r} \leq n, e_{\emptyset}=e_{0}=1$ and where the components $\alpha_{A}$ are real numbers. The conjugate of $\alpha$ is defined by $\bar{\alpha}=\sum_{A} \alpha_{A} \bar{e}_{A}$, with $\bar{e}_{A}=\bar{e}_{h_{r}} \bar{e}_{h_{r-1}} \ldots \bar{e}_{h_{1}} ; \bar{e}_{k}=$ $-e_{k}, k=1, \ldots, n, \bar{e}_{0}=e_{0}=1$.

In general, the vector space $\mathbb{R}^{n+1}$ is embedded in $\mathcal{C} \ell_{0, n}$ by identifying the element $\left(x_{0}, x_{1}, \ldots, x_{n}\right) \in \mathbb{R}^{n+1}$ with an element of the real vector space $\mathcal{A}_{n}:=\operatorname{span}_{\mathbb{R}}\left\{1, e_{1}, \ldots, e_{n}\right\} \subset \mathcal{C} \ell_{0, n}$. For our purpose we only consider such elements $x \in \mathcal{A}_{n}$ of the form $x=x_{0}+\sum_{k=1}^{n} e_{k} x_{k}=x_{0}+\underline{x}$, called paravectors (naturally, $x_{0}$ and $\underline{x}$ are called the scalar part and the vector part of $x$, respectively). Similarly to the complex case the conjugate $\bar{x}$ and the norm $|x|$ of $x$ are given by $\bar{x}=x_{0}-\underline{x}$ and $|x|=(x \bar{x})^{1 / 2}=(\bar{x} x)^{1 / 2}=\left(x_{0}^{2}+x_{1}^{2}+\right.$ $\left.\cdots+x_{n}^{2}\right)^{1 / 2}$, respectively.

In Hypercomplex Function Theory $\mathcal{C} \ell_{0, n}$-valued functions are studied. They are functions $f: \Omega \subseteq \mathbb{R}^{n+1} \cong \mathcal{A}_{n} \rightarrow \mathcal{C} \ell_{0, n}$ defined in an open subset by $f(z)=\sum_{A} f_{A}(z) e_{A}$, where $f_{A}(z)$ are real valued functions. We will focus on a special class of these functions analogous to complex holomorphic functions and connected with them via the following concept.

Definition 2.2. A function $f$ is called left (right) monogenic in $\Omega$ if it is a solution of the differential equation $\bar{\partial} f=0(f \bar{\partial}=0)$ where

$$
\bar{\partial}:=\frac{1}{2}\left(\partial_{0}+\partial_{\underline{x}}\right), \text { with } \partial_{0}:=\frac{\partial}{\partial x_{0}}, \text { and } \partial_{\underline{x}}:=\sum_{k=1}^{n} e_{k} \frac{\partial}{\partial x_{k}},
$$

generalizes the Cauchy-Riemann operator $\left(n=1, e_{1} \equiv i, z=x+i y\right)$

$$
\frac{\partial}{\partial \bar{z}}=\frac{1}{2}\left(\frac{\partial}{\partial x}+i \frac{\partial}{\partial y}\right)
$$

The operator $\partial:=\frac{1}{2}\left(\partial_{0}-\partial_{\underline{x}}\right)$ is called the conjugate generalized CauchyRiemann operator or the hypercomplex differential operator. 
Hereafter we only deal with left monogenic functions and we shall refer to them simply as monogenic functions (right monogenic functions are treated analogously). Notice that in the case of a paravector-valued function $f$ of the variable $x \in \mathcal{A}_{n}$, the hypercomplex partial differential equation $\bar{\partial} f=0$ is, except for the complex case $(n=1)$, equivalent to an over-determined

Cauchy-Riemann system of $\frac{n(n+1)+2}{2}$ first order differential equations for the $(n+1)$ component functions of $f$.

We remark that hypercomplex differentiability as generalization of complex differentiability has to be understood in the following way: a function $f$ defined in an open domain $\Omega \subseteq \mathbb{R}^{n+1}$ is hypercomplex differentiable supposed there exists in each point of $\Omega$ a uniquely defined areolar derivative $f^{\prime}$. Then $f$ is real differentiable and $f^{\prime}:=\partial f$. On the other hand, $f$ is hypercomplex differentiable in $\Omega$ if and only if $f$ is monogenic, cf. [19]. Consequently, if a hypercomplex function is monogenic then the existence of the hypercomplex derivative is guaranteed and can be obtained as result of the application of the conjugate generalized Cauchy-Riemann operator.

\section{Sequences of monogenic hypercomplex Appell polynomials}

Considering that for each $x \in \mathcal{A}_{n}$,

$$
\bar{\partial} x^{n}=\frac{1}{2}(1-n), \quad n \in \mathbb{N},
$$

only in the complex case $(n=1)$ the function $x^{n}$ belongs to the set of monogenic functions. Obviously, this fact causes problems for the consideration of monogenic polynomials in the ordinary way and, moreover, for the whole understanding of a suitable analog to power series in monogenic function theory, cf. [21]. Indeed, in some sense the problem of embedding integer powers of $x$ in a theory of monogenic functions was in the 90-ties of the last century for more than a decade a driving force for modifying or extending the class of monogenic functions. The result was, for example, the consideration of a Modified Clifford Analysis (H. Leutwiler et al.) or the introduction of holomorphic Cliffordian functions (G. Laville et al.). The third way to overcome the problem 'inside' of the class of ordinary monogenic functions, namely by generalizing Appell's concept of power-like polynomials will be explained now (for further details, see [9]).

Motivated by relation (2) and the previously mentioned concept of hypercomplex derivative, Appell sequences of homogeneous monogenic poly- 
nomials in the framework of Clifford Analysis have been introduced in the following way, [17, 24]:

Definition 3.1. A sequence of multivariate homogeneous polynomials $\left\{\phi_{k}(x)\right\}_{k \geq 0}$ of degree $k$ in the variable $x \in \mathcal{A}_{n}$ is called a generalized Appell sequence with respect to the hypercomplex differential operator $\partial$ if the following conditions are satisfied:

(i) $\bar{\partial} \phi_{k}(x)=0$, i.e., $\phi_{k}(x)$ is monogenic for each $k \geq 0$;

(ii) $\partial \phi_{k}(x)=k \phi_{k-1}(x), \quad k=1,2, \ldots$

Remark 3.2. Hereafter, if $x=x_{0}+\underline{x} \in \mathcal{A}_{n}$, we shall refer to $\phi_{k}(x)$ by using equivalently the following notations: $\phi_{k}\left(x_{0}+\underline{x}\right)$ or $\phi_{k}\left(x_{0}, \underline{x}\right)$.

Before generalizing the matrix approach to hypercomplex Appell polynomials, it is important to observe that, setting $p_{0}(x) \equiv c_{0} \neq 0$, from (2) the following explicit representation for the truncated sequence $\left\{p_{k}(x)\right\}_{k=0}^{m}$ of Appell polynomials in one real variable occurs:

$$
\begin{aligned}
p_{0}(x) & =c_{0} \\
p_{1}(x) & =c_{1}+c_{0} x \\
p_{2}(x) & =c_{2}+2 c_{1} x+c_{0} x^{2} \\
& \vdots \\
p_{m}(x) & =c_{m}+\left(\begin{array}{c}
m \\
1
\end{array}\right) c_{m-1} x+\left(\begin{array}{c}
m \\
2
\end{array}\right) c_{m-2} x^{2}+\cdots+\left(\begin{array}{c}
m \\
m
\end{array}\right) c_{0} x^{m},
\end{aligned}
$$

or, equivalently, in a more compact form

$$
p_{k}(x)=\sum_{j=0}^{k}\left(\begin{array}{c}
k \\
j
\end{array}\right) c_{j} x^{k-j}, \quad k=0,1, \ldots, m, \quad c_{0} \neq 0 .
$$

In particular, in this notation

$$
\mathbf{p}(0)=\left[\begin{array}{llll}
c_{0} & c_{1} & \ldots & c_{m}
\end{array}\right]^{T} .
$$

The formal replacement in (5) and (6) of the real variable $x$ by $x_{0}$, the scalar part of the paravector $x=x_{0}+\underline{x} \in \mathcal{A}_{n}$, as well as that of $c_{j}$ by $\tilde{c}_{j} \underline{x}^{j}$ leads to multivariate homogeneous polynomials

$$
\phi_{k}(x)=\sum_{j=0}^{k}\left(\begin{array}{c}
k \\
j
\end{array}\right) \tilde{c}_{j} \underline{x}^{j} x_{0}^{k-j}, \quad k=0,1, \ldots, m
$$


and to the vector $\phi(0, \underline{x})=\left[\begin{array}{llll}\tilde{c}_{0} \underline{x}^{0} & \tilde{c}_{1} \underline{x}^{1} & \ldots & \tilde{c}_{m} \underline{x}^{m}\end{array}\right]^{T}$. Consequently, recalling that the entries of the lower triangular generalized Pascal matrix $P\left(x_{0}\right) \equiv e^{H x_{0}}$ are given by

$$
\left(P\left(x_{0}\right)\right)_{i j}=\left\{\begin{array}{cl}
\left(\begin{array}{l}
i \\
j
\end{array}\right) x_{0}^{i-j}, & i \geq j \\
0, & \text { otherwise, } \quad i, j=0,1, \ldots, m,
\end{array}\right.
$$

and denoting by $\phi(x)=\left[\begin{array}{llll}\phi_{0}(x) & \phi_{1}(x) & \cdots & \phi_{m}(x)\end{array}\right]^{T}$, the corresponding matrix form of (7) is

$$
\phi(x)=e^{H x_{0}} \phi(0, \underline{x}),
$$

which represents the counterpart in the hypercomplex framework of (4). Introducing the diagonal matrix $D_{\tilde{c}}=\operatorname{diag}\left[\begin{array}{llll}\tilde{c}_{0} & \tilde{c}_{1} & \cdots & \tilde{c}_{m}\end{array}\right]$, and the vector

$$
\boldsymbol{\xi}(\underline{x})=\left[\begin{array}{lllll}
1 & \underline{x} & \underline{x}^{2} & \cdots & \underline{x}^{m}
\end{array}\right]^{T}
$$

the previous relation becomes

$$
\phi(x)=e^{H x_{0}} D_{\tilde{c}} \boldsymbol{\xi}(\underline{x}) .
$$

Now, the entries of such vector are generalized Appell polynomials with respect to $\partial$ if the two properties in Definition 3.1 are verified. It is evident that this could lead to impose some constraints on the diagonal coefficients of $D_{\tilde{c}}$. In order to deduce them, first of all we need to rewrite in matrix form the action of $\partial_{\underline{x}}$ on the vector $\boldsymbol{\xi}(\underline{x})$. By virtue to the fact that [15, p. 219]

$$
\partial_{\underline{x}}\left(\underline{x}^{k}\right)= \begin{cases}-k \underline{x}^{k-1}, & k \text { even } \\ -(n+k-1) \underline{x}^{k-1}, & k \text { odd }\end{cases}
$$

introducing the matrix $\tilde{H}$ defined by

$$
(\tilde{H})_{i j}= \begin{cases}-(n+i-1), & i=j+1 \wedge j \text { even } \\ -i, & i=j+1 \wedge j \text { odd } \\ 0, & \text { otherwise }, \quad i, j=0,1, \ldots, m\end{cases}
$$

we obtain

$$
\partial_{\underline{x}} \boldsymbol{\xi}(\underline{x})=\tilde{H} \boldsymbol{\xi}(\underline{x}),
$$

which means that $\tilde{H}$ is the derivation matrix playing the role of $\partial_{\underline{x}}$.

Remark 3.3. In the complex case $(n=1)$ we obtain simply $\tilde{H}=-H$. Denoting by $w=x_{0}+\underline{x}$, with $\underline{x}=i x_{1}$, it is easily checked that for $\boldsymbol{\xi}(w)=$ $\left[\begin{array}{lllll}1 & w & w^{2} & \cdots & w^{m}\end{array}\right]^{T}$ one has $\overline{\bar{\partial}} \boldsymbol{\xi}(w)=\mathbf{0}^{T}$, the null vector, and $\partial \boldsymbol{\xi}(w)=$ $H \boldsymbol{\xi}(w)$, i.e., the entries of $\boldsymbol{\xi}(w)$ are hypercomplex Appell polynomials. 
Therefore, by applying to both sides in (10) the hypercomplex differential operator we obtain

$$
\begin{aligned}
\bar{\partial} \phi(x) & =\frac{1}{2}\left(\partial_{0}+\partial_{\underline{x}}\right)\left(e^{H x_{0}} D_{\tilde{c}} \boldsymbol{\xi}(\underline{x})\right) \\
& =\frac{1}{2}\left[\left(\partial_{0} e^{H x_{0}}\right) D_{\tilde{c}} \boldsymbol{\xi}(\underline{x})+e^{H x_{0}} D_{\tilde{c}}\left(\partial_{\underline{x}} \boldsymbol{\xi}(\underline{x})\right)\right] \\
& =\frac{1}{2}\left[\left(H e^{H x_{0}}\right) D_{\tilde{c}} \boldsymbol{\xi}(\underline{x})+e^{H x_{0}} D_{\tilde{c}}(\tilde{H} \boldsymbol{\xi}(\underline{x}))\right] \\
& =\frac{1}{2} e^{H x_{0}}\left[H D_{\tilde{c}}+D_{\tilde{c}} \tilde{H}\right] \boldsymbol{\xi}(\underline{x}) .
\end{aligned}
$$

Remark 3.4. Notice that due to the uniqueness theorem for monogenic functions (cf. [22] or [18, p.180]) $D_{\tilde{c}}$ must be non-singular and we can suppose that all $\tilde{c}_{k} \neq 0, k=0,1, \ldots$ Otherwise the restriction of a component $\phi_{j}\left(x_{0}, \underline{x}\right)$ of $\phi(x)$ with $\tilde{c}_{j}=0$ to the hyperplane $x_{0}=0$ would have the value $\phi_{j}(0, \underline{x})=0$ and, consequently, be identically zero. But this contradicts the property of belonging to an Appell sequence.

We can now prove the following result.

Theorem 3.1. Suppose $\tilde{c}_{0} \neq 0$ is a given real number. The polynomials in (10) are monogenic if their remaining coefficients satisfy the conditions

$$
\tilde{c}_{2 k}=\tilde{c}_{2 k-1}=\frac{(2 k-1) ! !(n-2) ! !}{(n+2 k-2) ! !} \tilde{c}_{0}, \quad k=1,2, \ldots, n>1 .
$$

Proof. For $\phi(x)$ being monogenic, from (13) the diagonal matrix $D_{\tilde{c}}$ should be of such a form that

$$
H D_{\tilde{c}}+D_{\tilde{c}} \tilde{H}=O,
$$

the null matrix, which means that $H$ and $-\tilde{H}$ become similar matrices. By using the definitions of $H$ and $D_{\tilde{c}}$, the product $H D_{\tilde{c}}$ is given by

$$
\left(H D_{\tilde{c}}\right)_{i j}= \begin{cases}(j+1) \tilde{c}_{j}, & i=j+1 \\ 0, & \text { otherwise. }\end{cases}
$$

Similarly, from (11) the entries of $D_{\tilde{c}} \tilde{H}$ are

$$
\left(D_{\tilde{c}} \tilde{H}\right)_{i j}= \begin{cases}(n+j) \tilde{c}_{j+1}, & i=j+1 \wedge j \text { even } \\ (j+1) \tilde{c}_{j+1}, & i=j+1 \wedge j \text { odd } \\ 0, & \text { otherwise }\end{cases}
$$


Thus, the identity (15) is evident for $i \neq j+1$. For $(i=j+1 \wedge j$ odd) and $(i=j+1 \wedge j$ even $)$, one has

$$
(j+1) \tilde{c}_{j+1}=(j+1) \tilde{c}_{j}, \quad(n+j) \tilde{c}_{j+1}=(j+1) \tilde{c}_{j},
$$

respectively, or, equivalently,

$$
\tilde{c}_{2 k}=\tilde{c}_{2 k-1}, \quad \tilde{c}_{2 k-1}=\frac{2 k-1}{n+2 k-2} \tilde{c}_{2 k-2} .
$$

From these relations the assertion follows.

Remark 3.5. From (14) it is evident that the coefficients $\tilde{c}_{j}$ actually depends on $n$, i.e., $\tilde{c}_{j} \equiv \tilde{c}_{j}(n)$, for each $j \geq 1$.

Remark 3.6. The choice of the coefficients (14) with $\tilde{c}_{0}=1$ gives the generalized Appell sequence $\left\{\mathscr{P}_{k}^{(n)}(x)\right\}_{k \geq 0}$ introduced in 17 by a monogenic generating exponential function.

Under the hypothesis of Theorem 3.1 the polynomials $\phi_{k}(x)$ are monogenic. It remains to check if property (ii) in Definition 3.1 is satisfied. Applying (15) we get

$$
\begin{aligned}
\partial \phi(x) & =\frac{1}{2}\left[\left(\partial_{0}-\partial_{\underline{x}}\right)\left(e^{H x_{0}} D_{\tilde{c}} \boldsymbol{\xi}(\underline{x})\right)\right] \\
& =\frac{1}{2}\left[H e^{H x_{0}} D_{\tilde{c}} \boldsymbol{\xi}(\underline{x})-e^{H x_{0}} D_{\tilde{c}} \tilde{H} \boldsymbol{\xi}(\underline{x})\right] \\
& =\frac{1}{2} e^{H x_{0}}\left[H D_{\tilde{c}}-D_{\tilde{c}} \tilde{H}\right] \boldsymbol{\xi}(\underline{x})=H e^{H x_{0}} D_{\tilde{c}} \boldsymbol{\xi}(\underline{x})=H \boldsymbol{\phi}(x),
\end{aligned}
$$

which, actually, is the corresponding matrix representation of the property (ii) in Definition 3.1 ,

Remark 3.7. Following the recent article [11] on special properties of hypercomplex Appell polynomials like, for instance, three-term recurrence relations, further extensions including also all orthogonal Appell polynomial sequences obtained by the Gel'fand-Tsetlin procedure, cf. [6, 20], can be constructed.

In order to get such extensions, the suitable formal replacement in (5) and ([6) should be $x$ by $x_{0}$ and $c_{j}$ by $\tilde{c}_{j} \underline{x}^{j} Q_{s}(\underline{x})$, where $Q_{s}(\underline{x})$ is an arbitrary chosen monogenic polynomial of fixed degree $s=0,1,2, \ldots$.

One should notice that $Q_{s}(\underline{x})$ as an arbitrary chosen polynomial monogenic with respect to the generalized Cauchy-Riemann operator is automatically a generalized constant since it does not depend from $x_{0}$ and therefore 
belongs also to the kernel of the conjugated generalized Cauchy-Riemann operator. This fact implies that from the point of view of the hypercomplex derivative the polynomial $Q_{s}(\underline{x})$ behaves like an ordinary constant number, i.e., its hypercomplex derivative is constant zero. Moreover, one could come to the conclusion that the first polynomial of hypercomplex Appell sequences could be such an initial monogenic generalized constant $Q_{s}(\underline{x})$ and all polynomials of higher degree would have $Q_{s}(\underline{x})$ as common factor. This idea is realized in the paper [26].

The matrix form of these multivariate homogeneous polynomials is

$$
\phi(x)=Q_{s}(\underline{x}) e^{H x_{0}} D_{\tilde{c}} \boldsymbol{\xi}(\underline{x})
$$

and the constraints to be imposed on the diagonal entries of $D_{\tilde{c}}$ in order to get generalized Appell polynomials are

$$
\tilde{c}_{2 k}=\tilde{c}_{2 k-1}=\frac{(2 k-1) ! !(n+2 s-2) ! !}{(n+2 k+2 s-2) ! !} \tilde{c}_{0}, \quad k=1,2, \ldots, n>1 .
$$

Such constraints are achieved like in Theorem 3.1, but taking into account that the action of $\partial_{\underline{x}}$ on the vector $\boldsymbol{\xi}(\underline{x})$ is performed by the matrix $\tilde{H}^{s} Q_{s}(\underline{x})$, where

$$
(\tilde{H} s)_{i j}= \begin{cases}-(n+i+2 s-1), & i=j+1 \wedge j \text { even } \\ -i, & i=j+1 \wedge j \text { odd } \\ 0, & \text { otherwise, } \quad i, j=0,1, \ldots, m .\end{cases}
$$

This matrix results from the fact that

$$
\partial_{\underline{x}}\left(\underline{x}^{k} Q_{s}(\underline{x})\right)= \begin{cases}-k \underline{x}^{k-1} Q_{s}(\underline{x}), & k \text { even } \\ -(n+k+2 s-1) \underline{x}^{k-1} Q_{s}(\underline{x}), & k \text { odd } .\end{cases}
$$

\section{Special families of monogenic Appell polynomi- als}

We recall that the real Appell polynomials, $p_{k}(x)$, may also be characterized in terms of their generating exponential function of the form

$$
f(t) e^{t x}=\sum_{n=0}^{+\infty} p_{n}(x) \frac{t^{n}}{n !},
$$

where

$$
f(t)=\sum_{s=0}^{+\infty} c_{s} \frac{t^{s}}{s !}, \quad c_{0} \neq 0
$$


The creation matrix $H$ and formula (18) are the essential tools to get a transfer matrix whose action is to change the vector of monomial powers $\boldsymbol{\xi}(x)$ into the associated Appell vector $\mathbf{p}(x)$. Such matrix is $f(H)$ and it has been proved in [1, Theorem 3.2] that

$$
\mathbf{p}(x)=f(H) \boldsymbol{\xi}(x) .
$$

The generalized Appell sequence $\left\{\mathscr{P}_{k}^{(n)}(x)\right\}_{k \geq 0}$ referred in the Remark 3.6 allows to define a generalized exponential function in the hypercomplex setting by

$$
\operatorname{Exp}_{n}(x) \equiv e^{x_{0}} F(\underline{x})=\sum_{k=0}^{+\infty} \frac{\mathscr{P}_{k}^{(n)}(x)}{k !}, \quad x \in \mathcal{A}_{n},
$$

where

$$
F(\underline{x})=\sum_{s=0}^{+\infty} \tilde{c}_{s} \frac{\underline{x}^{s}}{s !}
$$

with $\tilde{c}_{0}=1$ and, for each $s \geq 1, \tilde{c}_{s}$ satisfying (14).

Due to the homogeneity of $\mathscr{P}_{k}^{(n)}(x)$, we have

$$
\operatorname{Exp}_{n}(t x)=\sum_{k=0}^{+\infty} \mathscr{P}_{k}^{(n)}(x) \frac{t^{k}}{k !},
$$

showing that the generating function of the Appell sequence $\left\{\mathscr{P}_{k}^{(n)}(x)\right\}_{k \geq 0}$ is of the form $G(x, t)=\operatorname{Exp}_{n}(t x), x \in \mathcal{A}_{n}, t \in \mathbb{R}$.

Remark 4.1. In the complex case, $G(x, t)=e^{t x}, x \in \mathbb{C}, t \in \mathbb{R}$ is the generating function of the basic Appell sequence $\left\{x^{k}\right\}_{k \geq 0}$. Thus, $\mathscr{P}_{k}^{(n)}(x)$ behave as monomial functions in the sense of the complex powers $w^{k}=$ $\left(x_{0}+i x_{1}\right)^{k}, k \geq 0$.

Using the hypercomplex exponential function (19) we define generalized Appell polynomials, in general non-homogeneous, as follows:

Definition 4.2. The sequence $\left\{\phi_{k}(x)\right\}_{k \geq 0}$ whose generating function is $G(x, t)=f(t) \operatorname{Exp}_{n}(t x), x \in \mathcal{A}_{n}$ and $f(t)$ a formal power series as in (18) is called generalized Appell sequence.

Noting that the function $f(t)$ coincides with the one appearing in the generating function of real Appell polynomials, the transfer matrix for $\phi(x), x \in$ $\mathcal{A}_{n}$, is also $f(H)$. However, in this case, $f(H)$ transforms the vector

$$
\boldsymbol{\xi}\left(\mathscr{P}^{(n)}(x)\right)=\left[\mathscr{P}_{0}^{(n)}(x) \mathscr{P}_{1}^{(n)}(x) \cdots \mathscr{P}_{m}^{(n)}(x)\right]^{T}
$$


in the generalized Appell vector, that is

$$
\phi(x)=f(H) \boldsymbol{\xi}\left(\mathscr{P}^{(n)}(x)\right) .
$$

Different kinds of generalized Appell polynomials can be derived by appropriate choice of $f(t)$. For instance, the corresponding transfer matrices for generalized Bernoulli, Frobenius-Euler, and monic Hermite polynomials are (see [1])

$$
\left(\sum_{k=0}^{m} \frac{H^{k}}{(k+1) !}\right)^{-1}, \quad(1-\lambda)(P-\lambda I)^{-1}, \quad e^{-H^{2} / 4}=\sum_{k=0}^{m} \frac{\left(-H^{2}\right)^{k}}{2^{2 k} k !},
$$

respectively. In particular, setting $\lambda=-1$ in the transfer matrix of generalized Frobenius-Euler polynomials, we get generalized Euler polynomials.

Remark 4.3. We notice that, when $\underline{x}=0$,

$$
\boldsymbol{\xi}\left(\mathscr{P}^{(n)}\left(x_{0}\right)\right) \equiv \boldsymbol{\xi}\left(x_{0}\right)
$$

while the components of $\phi\left(x_{0}\right)$ are the classical real Appell polynomials entries of the vector $\mathbf{p}\left(x_{0}\right)$.

\section{References}

[1] L. Aceto, H. R. Malonek, G. Tomaz, A unified matrix approach to the representation of Appell polynomials, Integral Transforms and Special Functions 26 (2015) 426-441.

[2] M. Anshelevich, Appell polynomials and their relatives III, Conditionally free theory, Illinois J. Math. 53 (2009) 39-66.

[3] P. Appell, Sur une classe de polynomes, Ann. Sci. Ècole Norm. Sup. 9 (2) (1880) 119-144.

[4] S. Bock, On monogenic series expansions with applications to linear elasticity, Adv. Appl. Clifford Algebras 24 (4) (2014) 931-943.

[5] S. Bock, K. Gürlebeck, On a generalized Appell system and monogenic power series, Math. Methods Appl. Sci. 33 (4) (2010) 394-411.

[6] F. Brackx, H. De Schepper, R. Lávička, V. Souček, Gel'fand-Tsetlin procedure for the construction of orthogonal bases in Hermitean Clifford analysis. In: T.E. Simos et al.(Eds.): Numerical Analysis and Applied Mathematics-ICNAAM 2010, AIP Conf. Proc. 1281, 2010, pp. 15081511. 
[7] I. Cação, D. Eelbode, Jacobi polynomials and generalized Clifford algebra-valued Appell sequences, Math. Methods Appl. Sci. 37 (2014) $1527-1537$.

[8] I. Cação, M. I. Falcão, H. R. Malonek, Laguerre derivative and monogenic Laguerre polynomials: an operational approach, Math. Comput. Modelling 53 (2011) 1084-1094.

[9] I. Cação, M. I. Falcão, H. R. Malonek, Matrix representations of a basic polynomial sequence in arbitrary dimension, Comput. Methods Funct. Theory 12 (2) (2012) 371-391.

[10] I. Cação, H. R. Malonek, On an hypercomplex generalization of Gould-Hopper and related Chebyshev polynomials. In: B. Murgante et al. (Eds.): Computational Science and Its Applications-ICCSA 2011 (LNCS 6784, Part III). Springer-Verlag, Berlin, 2011, pp. 316-326.

[11] I. Cação, M. I. Falcão and H. R. Malonek, Three-Term Recurrence Relations for Systems of Clifford Algebra-Valued Orthogonal Polynomials, 15p., doi:10.1007/s00006-015-0596-z

[12] F. Costabile, Expansions of real functions in Bernoulli polynomials and applications, Conf. Sem. Mat. Univ. Bari 273 (1999).

[13] F. Costabile, F. Dell'Accio, M.I. Gualtieri, A new approach to Bernoulli polynomials, Rendiconti di Matematica, Serie VII 26 (2006) 1-12.

[14] F. Costabile, E. Longo, A determinantal approach to Appell polynomials, J. Comp. Appl. Math. 234 (2010) 1528-1542.

[15] R. Delanghe, F. Sommen, V. Souček, Clifford Algebra and SpinorValued Functions. A function theory for the Dirac operator, Mathematics and its Applications (Dordrecht) 53 Kluwer Academic Publishers, 1992.

[16] M. I. Falcão, J. F. Cruz, H. R. Malonek, Remarks on the generation of monogenic functions. In: K. Gürlebeck, C. Könke (Eds.), $17^{\text {th }}$ Inter. Conf. on the Appl. of Computer Science and Mathematics in Architecture and Civil Engineering, Weimar, 2006, pp. 12-14.

[17] M. I. Falcão, H. R. Malonek, Generalized exponentials through Appell sets in $\mathbb{R}^{n+1}$ and Bessel functions. In: T. E. Simos, G. Psihoyios, C. Tsitouras (Eds.), AIP Conference Proceedings 936, 2007, pp. 738-741. 
[18] K. Gürlebeck, K. Habetha, W. Sprößig, Holomorphic Functions in the Plane and $n$-Dimensional Space, Translated from the 2006 German original. Birkhäuser Verlag, Basel, 2008.

[19] K. Gürlebeck, H. R. Malonek, A hypercomplex derivative of monogenic functions in $\mathbb{R}^{m+1}$ and its applications, Complex Variables 39 (1999) 199-228.

[20] R. Lávička, Complete orthogonal Appell systems for spherical monogenics, Complex Anal. Oper. Theory, 6 (2012) 477-489.

[21] H. R. Malonek, Power series representation for monogenic functions in $\mathbb{R}^{n+1}$ based on a permutational product, Complex Variables, Theory Appl. 15 (1990) 181-191.

[22] H. R. Malonek, in: S.-L. Eriksson (Eds.), Selected topics in hypercomplex function theory. In: Clifford algebras and potential theory, University of Joensuu, Research Reports 7, 2004, pp. 111-150.

[23] H. R. Malonek, M. I. Falcão, 3D-mappings by means of monogenic functions and their approximation, Math. Methods Appl. Sci. 33 (2010) 423-430.

[24] H. R. Malonek, M. I. Falcão, Special monogenic polynomials-properties and applications. In: T.E.Simos, G. Psihoyios, C. Tsitouras (Eds.), AIP Conference Proceedings 936, 2007, pp. 764-767.

[25] G. V. Milovanovic, Families of Euler-MacLaurin formulae for composite Gauss-Legendre and Lobatto quadratures, Bull., Cl. Sci. Math. Nat., Sci. Math. 145 (38) (2013) 63-81.

[26] D. Peña Peña, Shifted Appell Sequences in Clifford Analysis, Results. Math. 63 (2013) 1145-1157.

[27] P. E. Roman, T. Asahi, S. Casassus, Hermite-Gaussian functions for image synthesis, In: Asia-Pacific Signal and Information Processing Association, 2014 Annual Summit and Conference (APSIPA), 2014, pp. 1-9.

[28] P. Salminen, Optimal stopping, Appell polynomials, and Wiener-Hopf factorization, An International Journal of Probability and Stochastic Processes 83 (2011) 611-622. 
[29] R. M. Yamaleev, Pascal matrix representation of evolution of polynomials, Int. J. Appl. Comput. Math. 1 (4) (2015) 513-525.

[30] Y. Yang, H. Youn, Appell polynomials sequences: a linear algebra approach. JP Journal of Algebra, Number Theory and Applications 13 (2009) 65-98.

[31] St. Weinberg, The Quantum Theory of Fields, Cambridge University Press, Vol. 1, 1995. 